\title{
INFLUENCE OF HIGH SALINITY AND S-METHYLMETHIONINE ON SOME HEALTH-PROMOTING METABOLIC PROPERTIES OF GARDEN ROCKET LEAVES
}

\author{
LASZLO FODORPATAKI ${ }^{\mathrm{a}, \mathrm{b}, \mathrm{c}}$ *, MARTIN IAKAB ${ }^{\mathrm{c}}$, BERNAT TOMPA $^{\mathrm{a}}$
}

\begin{abstract}
Production of health-promoting substances in plants' metabolism may be modulated by treatment with chemical stress factors and natural bioactive compounds, which enhance stress tolerance through stimulation of biosynthesis of several protective metabolites. The aim of this work is to reveal metabolic interactions between high salinity stress (exerted by the presence of $100 \mathrm{mM} \mathrm{NaCl}$ in the mineral nutrient solution) and treatment of plants with $0.1 \mathrm{mM} \mathrm{S-methylmethionine} \mathrm{(also} \mathrm{known} \mathrm{as} \mathrm{vitamin} U$ ). This plantderived bioactive compound enhances the production of antioxidants such as carotenoids, ascorbate and other phenoloids in leaves of rocket plantlets exposed to salt stress, while reducing the concentration of toxic malondialdehyde and related catabolic products of membrane lipid peroxidation. Thus, a suitable combination of high salinity and plant hardening with S-methylmethionine may improve the quality of fresh rocket leaves upon human and animal consumption.
\end{abstract}

Keywords: ascorbate, carotenoids, phenoloids, lipid peroxidation, salt stress

\section{INTRODUCTION}

Treatment of crop plants with chemical stress factors and bioactive compounds modulates several metabolic processes, thus enhancing tolerance and resulting in accumulation of protective substances which possess healthpromoting qualities upon human consumption [1, 2, 3, 4]. Environmental stresses may elicit the biosynthesis of several metabolites in plants, including

a Babeş-Bolyai University, Faculty of Biology and Geology, 1 M. Kogalniceanu St., RO-400084, Cluj-Napoca, Romania

b Babeş-Bolyai University, Centre of Systemic Biology, Biodiversity and Bioresources, $1 \mathrm{M}$. Kogalniceanu St., RO-400084, Cluj-Napoca, Romania

c Sapientia Hungarian University of Transylvania, Department of Horticulture, 1C Sighisoarei Rd, RO-540485, Targu Mures, Romania

*Corresponding author: Ifodorp@gmail.com 
a wide range of bioactive molecules which contribute to the nutraceutical value of plant-derived foods. Beside controlled abiotic stressors, natural biostimulants can also be used to improve the quantity and nutritional quality of crops, by inducing directed metabolic changes in plant cell, with no need to genetically manipulate the plant material. Application of small amounts of biostimulants is also one of the most promising alternatives to cope with yield losses caused by environmental stress factors (such as drought, extreme temperatures, high salinity, soil contamination with heavy metals, pollutant gases in the atmosphere), excluding the extensive use of pesticides and artificial fertilizers $[5,6]$.

One of the most frequent chemical factors which may severely impair growth and yield of crop plants is salinity stress, caused by elevated sodium chloride concentration of the water in the soil which surrounds the root system. The short-term effect of high salinity is inhibition of growth, due to an osmotic imbalance related to water loss of root cells into the more concentrated solution of the rhizosphere. This can be compensated with osmotic adjustments, through up-regulation of the biosynthesis of certain compatible solutes, such as free proline, glycine betaine, sucrose, trehalose, sorbitol etc. This leads to the recovery of water balance of plant tissues but needs an input of extra energy for the antistress reactions [7, 8]. The main long-term effect of salt stress is ion toxicity induced by over-accumulation of the sodium ions in the cells, which leads to an imbalance in the homeostasis of inorganic ions needed for normal metabolic processes. This is related to the uptake antagonism of sodium with essential potassium and calcium ions, as well as to the inhibition of several enzymes which necessitate potassium ions for their proper functioning. The main reaction of plants to this ion toxicity is sequestration of sodium ions inside the vacuole, where no enzyme-catalyzed biochemical reactions occur $[9,10]$. A side effect of severe or prolonged salt stress is the accumulation of harmful reactive oxygen species (hydrogen peroxide, singlet oxygen, superoxide and hydroxyl radicals). This causes an oxidative stress, which can be counteracted by activation of the non-enzymatic and enzymatic constituents of the antioxidative defense system. As several antioxidants (e. g. vitamin C or ascorbic acid, carotenoids as precursors of vitamin A, vitamin E or tocopherol, certain flavones, flavonols, proanthocyanidines, polyphenols etc.) which accumulate in plants as a result of stress defense are appreciated as healthrelated compounds in the human diet, salt stress can be used for enhancing the nutritional quality of fresh vegetables $[11,12]$. Oxidative stress is generally associated with peroxidation of un-saturated fatty acids in membrane lipids, which affects selective membrane permeability and results in generation of toxic degradation products (mainly malondialdehyde), thus a specific component of stress tolerance is membrane protection against lipid peroxidation [13, 14]. 
Apart from adverse developmental conditions, plant metabolism can also be modulated by treatments with various bioactive phytochemicals. These compounds are non-nutrient plant molecules which trigger natural defense mechanisms, thus only small amounts are needed to promote growth, to enhance nutrient use efficiency, to improve crop productivity and to increase tolerance against stress factors. As modulators of physiological processes in plants, they may induce or enhance the biosynthesis of specific secondary metabolites (e. g. phenolic compounds through the shikimate pathway or terpenoids through the mevalonate pathway) to cope with stressful growth conditions, thus enriching vegetables and fruits in compounds with high nutraceutical value. As compared to complex product mixtures with a combination of several organic compounds (e. g. seaweed extracts, protein hydrolysates), pure organic active compounds present several advantages, since their optimal concentrations, physiological effects and action mechanisms can be determined more accurately. The most promising bioactive compounds which trigger metabolic defense against various abiotic stresses in plants are certain proteinogenic and non-proteinogenic amino acids, polyamines, oligopeptides, oligosaccharides and vitamins [3]. One of the less studied, but apparently very efficient natural bioactive compounds is Smethylmethionine, an amino acid derivative also known as vitamin $U[15,16]$. It is involved in important reactions of methylation related with detoxification of harmful organic pollutants and toxic metabolites, being ubiquitous in flowering plants. It increases tolerance of plants to extreme temperatures, to certain virus infections and to heavy metal toxicity, e. g. by stimulating the phenyl-propanoid pathway which provides plant cells with several protective molecules, including antioxidants and antimicrobial agents $[6,17,18,19]$.

Plant metabolites induced by environmental stress factors and by exogenous application of bioactive compounds exert their health-promoting effects especially upon consumption of fresh leafy vegetables in form of salads. Such vegetables include various cultivars of lettuce, spinach, artichoke, sorrel. At present, garden rocket (Eruca sativa), also known as rucola or arugula, is becoming a more and more popular leaf vegetable with a tart, bitter and peppery flavor. It is used for centuries in the Mediterranean and Asian traditional cuisine, but there is very scarce information about the impact of controlled growth conditions on its nutraceutical value [4, 20].

On the basis of the above-mentioned facts, the aim of this study is to reveal metabolic impacts of high salinity and of the application of Smethylmethionine, separately and in combination, on garden rocket plants and on the content of their leaves in organic compounds with relevance for human consumption, such as carotenoids and other photosynthetic pigments, phenolic compounds, ascorbic acid, products of membrane lipid peroxidation. 
Results of these investigations may contribute to the improvement of nutraceutical quality of the garden rocket as a healthy fresh vegetable.

\section{RESULTS AND DISCUSSION}

Carotenoids represent a large group of lipophilic plant pigments (tetraterpenes) with antioxidant properties, being able to annihilate such harmful reactive oxygen species as hydroxyl radicals, alkyl-peroxyl radicals and singlet oxygen [21]. Their amount in rocket leaves was significantly increased by exposure for one week to high salinity generated with $100 \mathrm{mM}$ $\mathrm{NaCl}$, and it was further elevated when salt stress was combined with addition of $0.1 \mathrm{mM} S$-methylmethionine to the aqueous solution used for watering the plantlets (Fig. 1). SMM applied under normal growth conditions did not cause any significant change in carotenoid content.

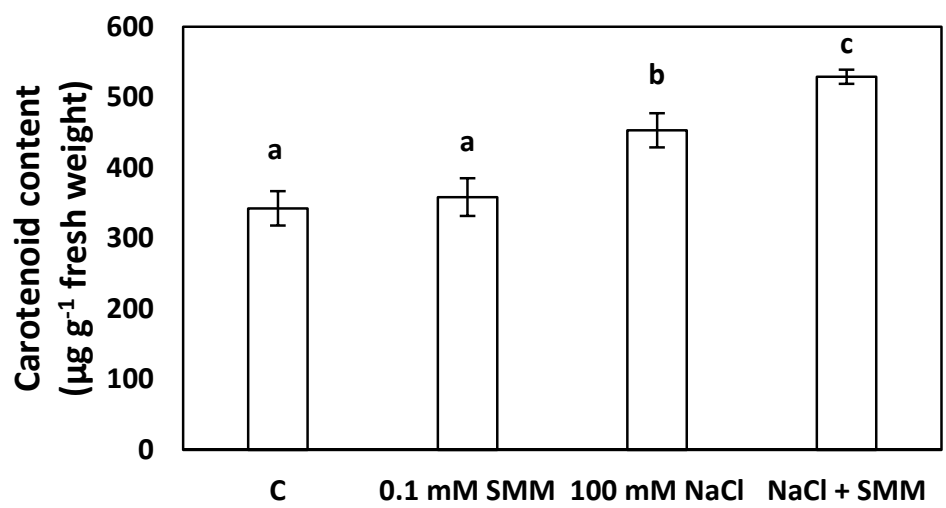

Figure 1. Influence of S-methylmethionine (SMM) and of high salinity, applied separately and in combination, on the carotenoid content of garden rocket leaves, after one week of treatment $(n=10$, vertical bars represent \pm SE from the means,

different letters indicate statistically significant differences at $P<0.05$ )

Stimulation of carotenoid synthesis by salt stress is most probably due to the oxidative burst generated by the persistence of high salinity for several days. When salt tolerance is developed by hardening, plants activate their antioxidative protection, and carotenoids represent a distinct category of nonenzymatic antioxidants, acting specifically in the photosynthetic apparatus located in the thylakoid membranes of chloro-plasts [22]. When stress tolerance becomes enhanced by exogenously supplied bioactive compounds, such as vitamin $\mathrm{U}(\mathrm{SMM})$, a more efficient antioxidative protection is associated 
with a further increment of the carotenoid pigment content of leaves. These results are in agreement with those reported by [4, 11], where enrichment of the irrigation water with $\mathrm{NaCl}$ increased the total carotenoid content (particularly the amounts of lutein and $\beta$-carotene) in lettuce leaves. Similar results were also reported for canola leaves, when plants were primed with vitamin $U$ before being exposed to low temperature stress [17]. Due to the fact that $\alpha$-carotene, $\beta$-carotene and $\beta$-cryptoxanthin are provitamin $A$ carotenoids that can be converted by the human body into vitamin $A$ (retinol), while many other carotenes (e. g. lycopene) and xanthophylls (e. g. lutein, zeaxanthin, antheraxantin) are effective antioxidants, their increased quantity enhances the health-promoting quality of rocket leaves in the human diet [3, 23].

In contrast with carotenoids, chlorophylls (being the main photosynthetic pigments of plants) may act as prooxidants, because upon absorption of light energy (especially under high photon flux densities) they lead to generation of singlet oxygen in the thylakoid membranes. Thus, a decreased ratio between prooxidant chlorophylls and antioxidant carotenoids confers a lowered risk of oxidative stress associated with metabolic imbalance due to unfavorable growth conditions [21]. While treatment of rocket plants with $0.1 \mathrm{mM} \mathrm{S}$ methylmethionine resulted in a moderate, but statistically significant increment of the chlorophylls to carotenoids ratio in leaves, salt stress induced for one week with $100 \mathrm{mM} \mathrm{NaCl}$ led to a decrement of this ratio from more than 3 to values approaching 2 (Fig. 2).

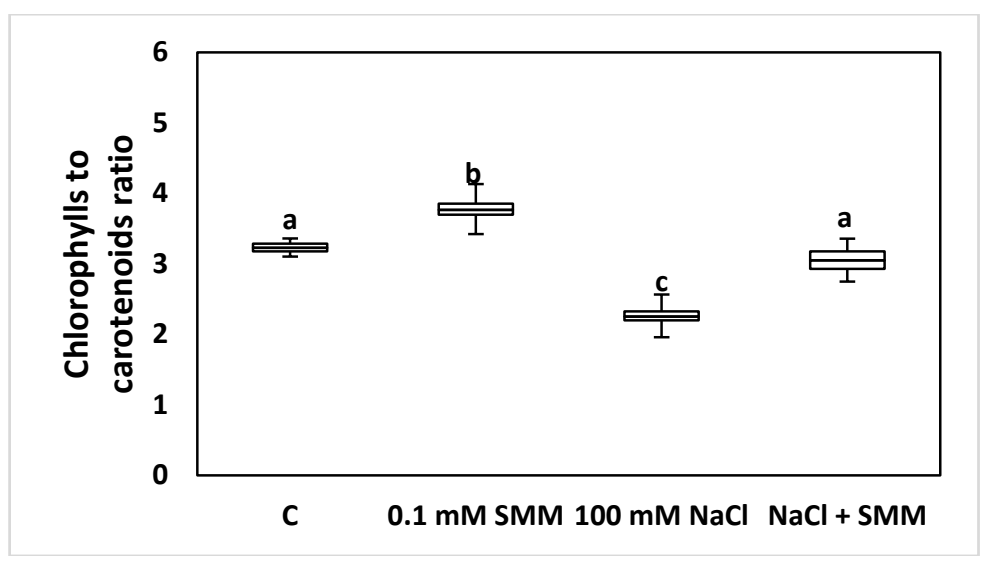

Figure 2. Changes of the chlorophylls to carotenoids ratio in leaves of rocket plants grown under constant light intensity and treated for one week with S-methylmethionine (SMM), high salt concentration and a combination of the former two chemical factors ( $n=10$, vertical bars represent $\pm S E$ from the means, different letters indicate statistically significant differences at $P<0.05$ ) 
When SMM treatment was applied to reduce the negative impact of high salinity, the chlorophylls to carotenoids ratio was restores to a level very close to the one that could be determined in the control plants. Because light intensity may considerably influence the chlorophyll and carotenoid pigment content of leaves, the plants were grown under constant photon flux density during the photoperiods. Increment of the chlorophylls to carotenoids ratio by SMM can be explained by the fact that this bioactive compound induced a moderately higher chlorophyll content of leaves (data not shown) but did not influence the amount of carotenoids. When SMM was applied simultaneously with salt stress, it totally annihilated the decreasing effect of high salinity on this ratio. Down-regulation of chlorophyll biosynthesis and up-regulation of chlorophyll degradation, as well as stimulation of carotenoid production as part of the antioxidative defense mechanism, was documented for several other crop plants, such as lettuce, broccoli, cauliflowers, tomato, pepper and melon fruits $[4,5,9]$. Interaction of SMM with salt stress on chlorophyll metabolism (in the porphyrin pathway) and on the metabolism of carotenoids (in the mevalonate pathway) is poorly documented in the literature. Similar results were reported when canola plants were treated with $1 \mathrm{mM} \mathrm{SMM} \mathrm{upon}$ exposure to two different salt concentrations (60 $\mathrm{mM}$ and $120 \mathrm{mM} \mathrm{NaCl}$ ) [24].

Phenoloids represent a large group of plant metabolites, many of them being produced as signaling molecules or defense effectors under various environmental stress conditions. Their biosynthesis in plants proceeds through the shikimate pathway, and in some cases the malonate pathway also has a contribution to it. By modulation of these biosynthetic pathways, plant exhibit a metabolic plasticity that results in increased tolerance to adverse developmental conditions. The main groups of plant phenolic compounds are water-soluble simple phenoloids (e. g. coumarins), flavonoids (anthocyanins, flavones, flavonols, flavanols, flavanones, isoflavones), hydrolysable tannins, as well as complex tannins and condensed phenylpropanoids, which are not soluble in water. Several different representatives have antioxidant properties, while others exert other health-protective effects upon ingestion. In plants, their aromatic rings also confer protection against ultraviolet radiations [9, 26]. This is the reason why the variation of overall phenoloid content of rocket leaves was selected as a possible biochemical marker of tolerance against the oxidative stress associated with high salinity, as well as of the benefic influence of vitamin $U$ on protective processes. After seven days of treatment, $0.1 \mathrm{mM}$ SMM increased the phenoloid content of rocket leaves by about $20 \%$ (Fig. 3). This increment was even more pronounced upon long-term salt stress, while the highest phenoloid content (approximately two times higher than in the control) was measured when high salinity was combined with SMM treatment. 


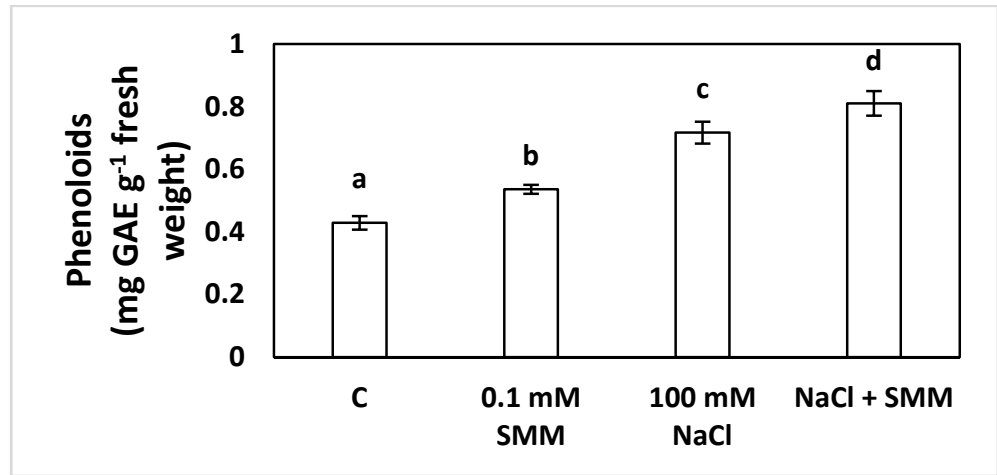

Figure 3. Phenoloid content of garden rocket leaves, expressed as gallic acid equivalents (GAE), upon exposure for seven days to $0.1 \mathrm{mM}$ S-methylmethionine (SMM), to high salinity induced with $100 \mathrm{mM} \mathrm{NaCl}$, and to the combination of the former two chemical agents $(n=10$, vertical bars represent $\pm S E$ from the means, different letters indicate statistically significant differences at $P<0.05$ )

Experiments performed with maize plants exposed to low temperature have shown that the phenylpropanoid pathway is stimulated by $\mathrm{S}$ methylmethionine [6]. This bioactive compound also induced an increased phenoloid content of lettuce leaves exposed for six days to $75 \mathrm{mM}$ and 150 $\mathrm{mM} \mathrm{NaCl}$ but did not cause any significant increment in the overall phenolic compound content if salt stress was imposed for a shorter period of time (three days instead of six days) [11]. These data are in agreement with those experiments which demonstrated that phenolic compounds accumulate under stress conditions later than other non-enzymatic antioxidants, being responsible mainly for the reestablishment of redox homeostasis after prolonged exposure to adverse conditions, when the other ways of antioxidative defense have become weakened [10, 28, 29]. It was also demonstrated that the highest antioxidant capacity is possessed by phenolic compounds with several hydroxide groups linked to the aromatic ring $[29,30]$.

The ascorbic acid (vitamin C) content, which represents a major indicator of health-promoting quality of plant materials, was not significantly influenced by S-methylmethionine, but when this bioactive compound was supplied to salt-stressed rocket plants, the vitamin C content of leaves was increased by about $30 \%$ (Fig. 4). The long-term exposure of plantlets to high salinity also led to a statistically significant elevation of ascorbate content, and this increment was further intensified by SMM, probably as a result of stimulation of antioxidative protection upon the installation of oxidative stress conditions associated with sustained salt stress. 


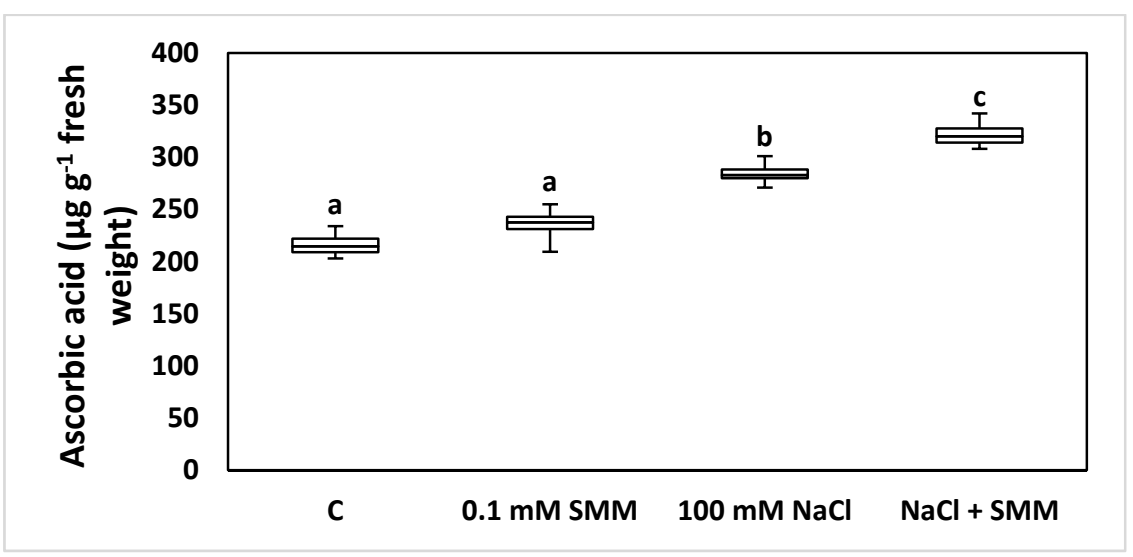

Figure 4. Variations in the ascorbic acid content of garden rocket leaves after seven days of treatment with $0.1 \mathrm{mM}$ S-methylmethionine (SMM), with $100 \mathrm{mM}$ $\mathrm{NaCl}$, and with the combination of the former two chemical agents $(n=10$, vertical bars represent $\pm S E$ from the means, different letters indicate statistically significant differences at $P<0.05$ )

In lettuce leaves $0.25 \mathrm{mM}$ and $2 \mathrm{mM}$ SMM moderately increased the vitamin $\mathrm{C}$ content and compensated for its significant reduction by development at $5^{\circ} \mathrm{C}$ [17]. Priming of lettuce plantlets with $0.05 \mathrm{mM} \mathrm{SMM}$ for three days prior to exposure to salt stress resulted in an increment of ascorbate content until the third day of salt treatment, and remained stabilized at this higher level during the following three days [11]. These results suggest that SMM may enhance the antioxidative defense of plants through the triggering of an up-regulation of ascorbate biosynthesis under adverse growth conditions, such as high salinity and low temperature. Increased ascorbic acid quantities were also reported in leaves of five weeks old lettuce plants exposed to chilling stress and heat shock, a positive correlation between the overproduction of hydrogen peroxide and vitamin C content being established [26]. In soybean varieties differing in flavonoid content increased levels of UV-B radiation decreased ascorbate content and increased dehydroascorbate content, in relation with an enhanced oxidative stress [30]. It was also shown that under the influence of various inhibitors of enzymatic browning in lettuce leaves, increased ascorbic acid content protected phenolics from oxidation [28]. Thus, it seems that vitamin $C$ content of leaves can be increased by exposure for several days to mild stress conditions and by application of bioactive compounds, especially when these are used simultaneously with or prior to stress factors. This increases the nutritional value of freshly consumed vegetables. 
Whenever metabolic impairments result in over-accumulation of reactive oxygen species, oxidative membrane damage occurs. This affects the structural and functional properties of membrane lipide and membraneassociated proteins, leading to the loss of selective permeability of membranes and to toxic products of peroxidation of unsaturated fatty acids present in membrane lipids [31]. Highly toxic products of lipid peroxidation, referred to as thiobarbituric acid-reactive substances (TBARS), the mostly dangerous one being malondialdehyde, were generated in increased amounts in the living cells of rocket plants exposed for seven days to $100 \mathrm{mM} \mathrm{NaCl}$ (Fig. 5). This indicates that long-term exposure to high salinity causes oxidative stress which damages biomembranes.

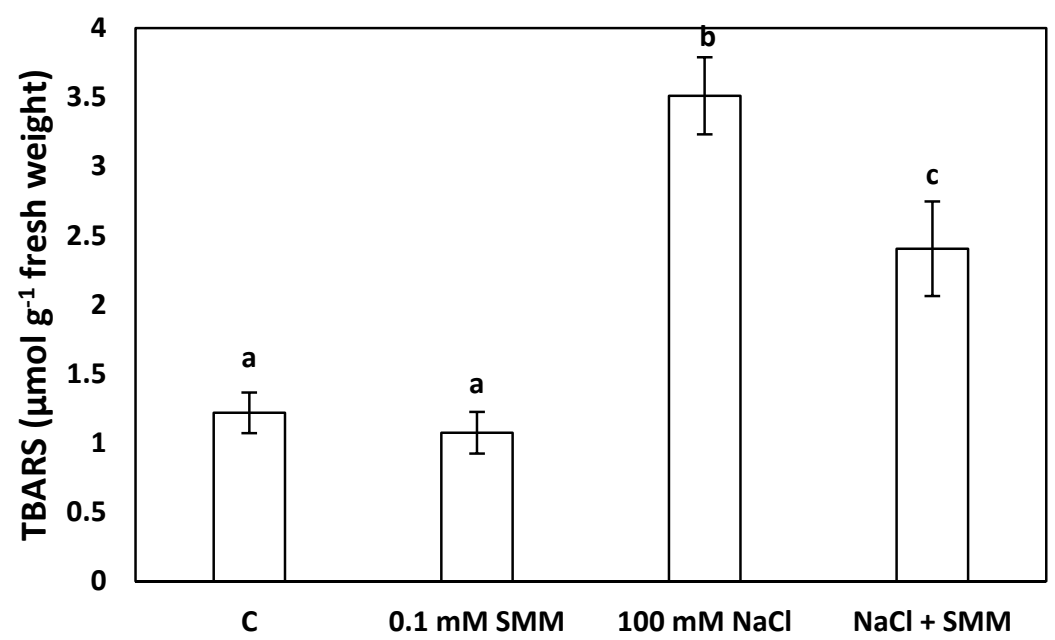

Figure 5. Influence of high salinity and treatment with S-methylmethionine (SMM) on the amount of thiobarbituric acid-reactive substances (TBARS) resulted from oxidative damage of membrane lipids in rocket plants $(n=10$, vertical bars represent $\pm S E$ from the means, different letters indicate statistically significant differences at $P<0.05$ )

The generated TBARS may damage proteins and nucleic acids in plants as well as in humans, upon consumption of plants as part of the diet. This is one reason why it is important to reduce the TBARS content of crop plants grown under harmful conditions. This can be achieved by supplying $0.1 \mathrm{mM} \mathrm{SMM}$ to salt-stressed plants, the treatment being able to significantly reduce the toxic TBARS content. Because SMM did not induce oxidative stress, it did not cause changes in the very low TBARS content characteristic for the unstressed control plants. But it significantly diminished the TBARS 
production in salt-stressed plants (Fig. 5), thus reducing the amount of undesirable toxic products of lipid peroxidation. These results suggest that SMM may enhance the capacity of the enzymatic and/or non-enzymatic components of the antioxidative protection system to reduce the concentration of reactive oxygen species generated due to metabolic imbalances. Similar results were obtained when canola plants were grown on substrates with different salt concentrations. The degree of oxidative membrane damage was proportional with the level of salinity, being lower at $60 \mathrm{mM} \mathrm{NaCl}$ than at $120 \mathrm{mM}$ $\mathrm{NaCl}$. Exogenously applied SMM (1 mM, by leaf spraying) totally annihilated the increment of TBARS production generated by $60 \mathrm{mM} \mathrm{NaCl}$ and partly reduced the concentration of toxic products of lipid peroxidation when plants were exposed to a more severe salt stress induced with $120 \mathrm{mM} \mathrm{NaCl}$ [24]. Increased lipid peroxidation was also reported when plants were exposed to soil pollution with water-soluble forms of various heavy metals, to intense UV-B radiations, to air pollution with ozone and nitrogen oxides, to severe drought or to extreme temperatures [5, 13, 31, 33].

\section{CONCLUSIONS}

High salinity stress caused by the presence of $100 \mathrm{mM} \mathrm{NaCl}$ in the aqueous solution around the root system, increased carotenoid content, total phenoloid content, ascorbic acid content and the amount of toxic derivatives of lipid peroxidation in young leaves of garden rocket plants. S-methylmethionine or vitamin $U$, as a natural metabolic product of plants, applied exogenously in the concentration of $0.1 \mathrm{mM}$, acting as a bioactive compound that counteracts negative effects of salt stress, induced a further increment in the carotenoid pigment, phenoloid and ascorbic acid content, thus contributing to an enhanced antioxidative protection of leaf cells. Furthermore, this amino acid derivative reduced the production of malondialdehyde and other thiobarbituric acid-reactive substances generated upon oxidative damage of membrane lipids. All these metabolic changes increased the health-promoting quality of fresh rocket leaves upon human consumption.

This is a novel contribution to cost-effective modulation of biochemical processes in the metabolism of garden rocket plants, in order to increase the content of certain antioxidants (carotenoids, phenoloids, ascorbic acid) in leaves, and to annihilate negative effects of high salinity with the use of a plant-derived bioactive compound (S-methylmethionine), thus avoiding pesticides and genetic manipulations in the attempt to improve yield and nutraceutical quality of this leafy vegetable. 


\section{EXPERIMENTAL SECTION}

Garden rocket or "rucola" (Eruca sativa Mill.) belongs to the cabbage and mustard family (Brassicaceae), possessing a specific metabolic pathway for the synthesis of sulfur-containing glucosinolates. Because they confer a peppery flavor to the leaves, rocket plants are widely used as salad vegetables in the health-promoting human diet. Garden rocket seeds were germinated in pots with perlite as a soil substitute, and plantlets were watered twice a week with Hoagland's mineral nutrient solution [11], being grown in an environmental chamber (Sanyo MLR-351H) under a photosynthetically active photon flux density of $330 \mu \mathrm{mol} \mathrm{m}^{-2} \mathrm{~s}^{-1}$ for a daily photoperiod of $14 \mathrm{~h}$, at $22^{\circ} \mathrm{C}$ in the light period and $18^{\circ} \mathrm{C}$ in the dark period, the relative air humidity being maintained at $70 \%$. Three weeks old plantlets were exposed for seven days to different salt and triacontanol treatments: control plants were watered with Hoagland's basal solution, salt-stressed plants were subjected to the presence of $100 \mathrm{mM} \mathrm{NaCl}$ dissolved in the nutrient solution, treatment with vitamin $U$ was achieved with $0.1 \mathrm{mM}$ S-methylmethionine (SMM) added to the nutrient medium, while salt-stressed and vitamin U-treated plants received the mineral nutrient medium with the simultaneous addition of $100 \mathrm{mM} \mathrm{NaCl}$ and $0.1 \mathrm{mM}$ SMM. The concentrations were selected from previous experiments $(100 \mathrm{mM} \mathrm{NaCl}$ induces a moderate salt stress in rocket plants, and $0.1 \mathrm{mM}$ is a concentration of SMM which does not exert any detectable negative effect during early developmental stages of plants). Each of the four experimental variants was set in ten separate repetitions, with one plant per pot. Parts of the second fully expanded leaf from the top of the stem were used for biochemical determinations.

The quantity of photosynthetic pigments (chlorophyll $a$, chlorophyll $b$ and carotenoids) was determined photometrically (with a Jasco V750 UV-Vis spectrophotometer). In a pre-chilled mortar $0.25 \mathrm{~g}$ fresh weight of leaves were homogenized in $5 \mathrm{~mL}$ of $80 \%(\mathrm{v} / \mathrm{v})$ acetone. The supernatant resulting from centrifugation of the acetonic extract for $10 \mathrm{~min}$ at $4000 \mathrm{~g}$ was used for absorbance measurements at the wavelengths of $663 \mathrm{~nm}, 646 \mathrm{~nm}$ and $470 \mathrm{~nm}$. The reference sample consisted of acetone $80 \%$. The quantities of chlorophyll $a$, chlorophyll $b$ and carotenoid pigments were determined based on the absorbance values measured at the three above-mentioned wavelengths, being expressed as mg per gram leaf fresh weight, according to [22].

The total phenolics content of rocket leaves was determined photometrically, using the Folin-Ciocâlteu reagent method $[25,26] .1 \mathrm{~g}$ fresh leaf was homogenized in a mortar with $6 \mathrm{~mL} 80 \%(\mathrm{v} / \mathrm{v})$ acetone, the mixture was incubated in darkness at $4^{\circ} \mathrm{C}$ overnight, then it was centrifuged at $2000 \mathrm{~g}$ for $3 \mathrm{~min}$. $0.2 \mathrm{~mL}$ of supernatant was supplemented with $1 \mathrm{~mL}$ of $10 \%(\mathrm{v} / \mathrm{v})$ Folin- 
Ciocâlteu reagent. After $5 \mathrm{~min}, 0.8 \mathrm{~mL}$ of $7.5 \%$ (w/v) sodium carbonate was added to the previous mixture. After incubation in the dark at $45^{\circ} \mathrm{C}$ for $15 \mathrm{~min}$, the absorbance of the resulting blue solution was measured at $765 \mathrm{~nm}$ (with a Jasco V750 UV-Vis spectrophotometer). A blank was prepared with $0.2 \mathrm{~mL}$ of $80 \%(\mathrm{v} / \mathrm{v})$ acetone. A gallic acid standard curve was prepared from a freshly made stock solution of $1 \mathrm{mg} \mathrm{mL}^{-1}$ gallic acid in $80 \%(\mathrm{v} / \mathrm{v})$ acetone, and the total phenoloid content of leaves was expressed in gallic acid equivalents on a fresh weight basis [27, 30].

Extraction and determination of the ascorbic acid content of rocket leaves was performed according to [32]. $0.5 \mathrm{~g}$ of freshly harvested leaf blades were homogenized in a prechilled mortar with $4 \mathrm{~mL}$ of $6 \%(\mathrm{w} / \mathrm{v})$ trichloroacetic acid. The mixture was centrifuged at $16500 \mathrm{~g}$ and $4^{\circ} \mathrm{C}$ for $15 \mathrm{~min}$, and $0.2 \mathrm{~mL}$ of supernatant was supplemented with a mixture of trichloroacetic acid, ortophosphoric acid, dithiothreitol, ferric chloride, N-ethylmaleimide, ethanolic solution of 2,2'-dipyridyl, all of these being dissolved in sodium phosphate buffer ( $\mathrm{pH} \mathrm{7.4).} \mathrm{After} \mathrm{one} \mathrm{hour} \mathrm{of} \mathrm{incubation} \mathrm{at} 42^{\circ} \mathrm{C}$ the absorbance of the solution was measured at $525 \mathrm{~nm}$ with a spectrophotometer. The oxidized form of vitamin $\mathrm{C}$ (dehydroascorbate) was converted by dithiothreitol into the reduced form, thus after incubation the total ascorbic acid content of samples was present in its reduced state. This reduced form of ascorbic acid generated ferro ions from the ferric chloride, and the ferro ions gave a colored product with 2,2'-dipyridyl. The quantity of ascorbic acid in the leaf samples was determined through a calibration curve obtained with known concentrations of pure ascorbic acid [31, 32].

Oxidative membrane damage was evaluated through the generation of malondialdehyde and other related thiobarbituric acid-reactive substances (TBARS) because of peroxidation of unsaturated fatty acids from the lipid bilayer of cell membranes [2, 14]. $0.5 \mathrm{~g}$ fresh weight of leaf blades were homogenized in a pre-chilled mortar with $5 \mathrm{~mL}$ of $0.1 \%(\mathrm{w} / \mathrm{v})$ trichloroacetic acid. The homogenate was centrifuged at $15000 \mathrm{~g}$ for $15 \mathrm{~min}$, then $2 \mathrm{~mL}$ of the supernatant was supplemented with $4 \mathrm{~mL}$ of $10 \%(\mathrm{w} / \mathrm{v})$ trichloroacetic acid in which $0.5 \%(\mathrm{w} / \mathrm{v}) 2$-thiobarbituric acid was dissolved previously. The mixture was heated at $95^{\circ} \mathrm{C}$ for $30 \mathrm{~min}$, then cooled in an ice bath. The solution that turned light red was centrifuged at $10000 \mathrm{~g}$ for $5 \mathrm{~min}$ and the absorbance of the supernatant was measured at $532 \mathrm{~nm}$ and $600 \mathrm{~nm}$. The value obtained at 600 $\mathrm{nm}$ (related to the presence of interfering anthocyanins) was deduced from the one measured at $532 \mathrm{~nm}$. TBARS concentration of the leaf samples was calculated using the extinction coefficient of $155 \mathrm{mM}^{-1} \mathrm{~cm}^{-1}[14,31]$. 
Experiments were performed with ten plants for each treatment, while measurements had three technical repetitions for every plant material. The $R$ statistical package (R Core Team 2019) was used for statistical data analyses. Normality of data distribution was evaluated with the Shapiro-Wilk test, and Bartlett's test was used for analysis of the homogeneity of variances. Data were represented as the mean \pm standard error (SE). Significant differences were determined with the one-way ANOVA and the post-hoc Tukey HSD test, differences being considered significant at $P<0.05$.

\section{ACKNOWLEDGMENTS}

This research was partly supported by the Institute of Advanced Studies in Science and Technology of the "Babeș-Bolyai" University in Cluj-Napoca, Romania, through a fellowship for excellence in research, awarded in 2020 to M. Sc. student Bernat Tompa.

\section{REFERENCES}

1. R. Bulgari; G. Franzoni; A. Ferrante; Agronomy, 2019, 9, 306-316

2. P. du Jardin; L. Xu; D. Geelen; Agricultural functions and action mechanisms of plant biostimulants (PBs). In The Chemical Biology of Plant Biostimulants, D. Geelen; L. Xu, Eds.; Wiley Online Books, Hoboken, USA, 2020, Chapter 1, pp. 1-30

3. A.L. Garcia-Garcia; F.J. Garcia-Machado; A.A. Borges; S. Morales-Sierra; A. Boto; D. Jimenez-Arias; Front. Plant Sci., 2020, 11, 575829

4. S. Toscano; A. Trivellini; G. Cocetta; R. Bulgari; A. Francini; D. Romano; A. Ferrante; Front. Plant Sci., 2019, 10, 1212

5. C. Kaya; M. Ashraf; O. Sonmez; A.L. Tuna; T. Polat; S. Aydemir; Acta Physiol. Plant., 2015, 37, 1729-1741

6. K. Paldi; I. Racz; Z. Szigeti; S. Rudnoy; Biol. Plant., 2014, 58, 189-194

7. E. Blumwald; Curr. Opin. Cell Biol., 2000, 12, 431-434

8. D. Rhodes; A. Nadolska-Orczik; P.J. Rich; Salinity, osmolytes and compatible solutes. In Salinity:Environment-Plant-Molecules, A. Lauchli; U. Luttge, Eds.; Kluwer Academic Publishers, Dordrecht, the Netherlands, 2002, pp. 181-204

9. C. Ouhibi; H. Attia; F. Rebah; N. Msilini; M. Chebbi; J. Aarrouf; L. Urban; M. Lachaal; Plant Physiol. Biochem., 2014, 83, 126-133

10. C. Sgherri; U. Perez-Lopez; F. Micaelli; J. Miranda-Apodaca; A. Mena-Petite; A. Munoz-Rueda; M. F. Quartacci; Plant Physiol. Biochem., 2017, 115, 269-278

11. L. Fodorpataki; B. Holinka; E. Gyorgy; Priming with S-methylmethionine increases non-enzymatic antioxidant content of lettuce leaves exposed to salt stress. In Controlled Environment Agriculture, M. Asaduzzaman, Ed.; Nova Science Publishers, New York, USA, 2016, Chapter 6, pp. 133-164

12. J. Zhu; Trends Plant Sci., 2001, 6, 66-72 
13. A. Baryla; C. Laborde; J.-L. Montillet; C. Triantaphylides; P. Chagvardieff; Env. Poll., 2000, 109, 131-135

14. N. Jambunathan; Meth. Mol. Biol., 2010, 639, 291-298

15. A.D. Patel; N.K. Prajapati; J. Chem. Pharm. Res., 2012, 4, 209-215

16. P. Ranocha; S.D. McNeil; M.J. Ziemak; C. Li; M.C. Tarczynski; A.D. Hanson; Plant J., 2001, 25, 575-584

17. L. Fodorpataki; K. Molnar; B. Tompa; S.R.C. Plugaru; Not. Bot. Horti Agrobot., 2019, 47, 592-598

18. E. Ludmerszki; K. Paldi; I. Racz; Z. Szigeti; S. Rudnoy; Appl. Ecol. Environ. Res., 2014, 12, 777-785

19. C. Olah; E. Ludmerszki; I. Racz; G. Balassa, S. Rudnoy; Russ. J. Plant Physiol., 2018, 65, 63-68

20. G. Cocetta; S. Mishra; A. Raffaelli; A. Ferranta; J. Plant Physiol., 2018, 231, 261-270

21. N. Smirnoff; Ascorbate, tocopherol and carotenoids: metabolism, pathway engineering and functions. In Antioxidants and Reactive Oxygen Species in Plants, N. Smirnoff, Ed.; Blackwell Publishers, Oxford, United Kingdom, 2005, pp. 53-86

22. H.K. Lichtenthaler; A.R. Wellburn; Biochem. Soc. Trans., 1983, 11, 591-592

23. S.S. Gill; N. Tuteja; Plant Physiol. Biochem., 2010, 48, 909-930

24. L. Fodorpataki; K. Molnar; B. Tompa; C. Bartha; Intl. J. Agric. Biol., 2021, 25, 11-19

25. Z. Louail; N. Djemouai; S. Krimate; K. Bouti; S. Bouti; H. Tounsi; A. Kameli; Anal. Univ. Oradea, 2020, 27, 215-223

26. M.-M. Oh; E.E. Carey; C.B. Rajashekar; Plant Physiol. Biochem., 2009, 47, 579583

27. V. Mulabagal; M. Ngouajio; A. Nair; Y. Zhang; A.L. Gottumukkala; M.G. Nair; Food Chem., 2010, 118, 300-306

28. A. Altunkaya; V. Gokmen; Food Chem., 2008, 107, 1173-1179

29. R. Llorach; A. Martinez-Sanchez; F.A. Tomas-Barberan; M.I. Gil; F. Ferreres; Food Chem., 2008, 1028-1038

30. V.L. Singleton; R. Orthofer; R.M. Lamuela-Raventos; Meth. Enzymol., 1999, 299, 152-178

31. S.K. Panda; I. Chaudhury; M.H. Khan; Biol. Plant., 2003, 46, 289-294

32. K. Kampfenkel; M. van Montagu; D. Inze; Anal. Biochem., 1995, 225,165-167

33. C. Xu; S. Natarajan; J.H. Sullivan; Env. Exp. Bot, 2008, 63, 39-48

34. K. Molnar; B. Biro-Janka; I.I. Nyaradi; L. Fodorpataki; B.E. Varga; J. Balint; M.M. Duda; Acta Biol. Maris., 2020, 3, 48-55 